\title{
Erratum: ReFlexLab: Designing Transitive Wearable Technologies towards Poetic Aesthetics
}

\author{
Clemens Winkler ${ }^{1}$ and Soomi Park ${ }^{2}$ \\ ${ }^{1}$ Zurich University of the Arts, Interaction Design, Ausstellungsstr, \\ 60, 8031 Zurich, Switzerland \\ clemens.winkler@zhak.ch \\ ${ }^{2}$ Royal College of Art, Design Interactions, Kensington Gore, \\ SW7 2EU, London, United Kingdom \\ soomi.park@network.rca.ac.uk
}

DOI 10.1007/978-3-319-07635-5_70

The paper starting on page 731 of this volume has been retracted as text was taken verbatim from the paper "UISilk: towards interfacing the body" by Veronica Ranner, published in the proceedings of SMI'13, (C) ACM, NY, 2013. 